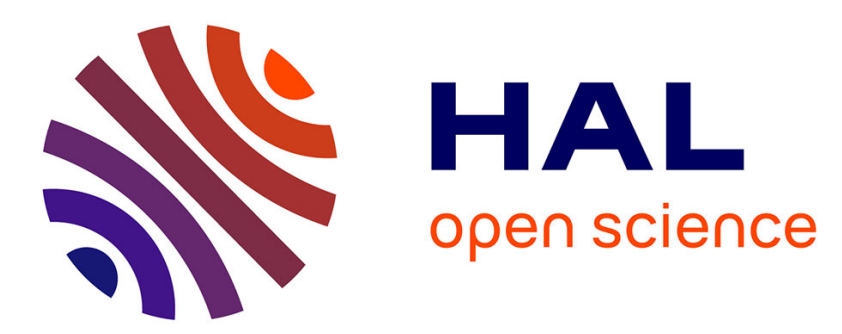

\title{
Experimental Study Of The Interactions Betwen Air Flow Rate Modulations And PVC In A Swirl-Stabilised Liquid Fuel Burner
}

Antoine Renaud, Sebastien Ducruix, Philippe Scouflaire, Laurent Zimmer

\section{- To cite this version:}

Antoine Renaud, Sebastien Ducruix, Philippe Scouflaire, Laurent Zimmer. Experimental Study Of The Interactions Betwen Air Flow Rate Modulations And PVC In A Swirl-Stabilised Liquid Fuel Burner. Proceedings of ASME Turbo Expo 2015 GT2015, Jun 2015, Montreal, Canada. $10.1115 /$ gt2015-42775 . hal-01286619

\section{HAL Id: hal-01286619 https://hal.science/hal-01286619}

Submitted on 28 Feb 2020

HAL is a multi-disciplinary open access archive for the deposit and dissemination of scientific research documents, whether they are published or not. The documents may come from teaching and research institutions in France or abroad, or from public or private research centers.
L'archive ouverte pluridisciplinaire HAL, est destinée au dépôt et à la diffusion de documents scientifiques de niveau recherche, publiés ou non, émanant des établissements d'enseignement et de recherche français ou étrangers, des laboratoires publics ou privés. 


\title{
Proceedings of ASME Turbo Expo 2015: Turbine Technical Conference and Exposition
}

June 15-19, 2015, Montréal, Canada

\section{EXPERIMENTAL STUDY OF THE INTERACTIONS BETWEEN AIR FLOW RATE MODULATIONS AND PVC IN A SWIRL-STABILISED LIQUID FUEL BURNER}

\author{
Antoine Renaud, Sébastien Ducruix, Philippe Scouflaire, Laurent Zimmer \\ ${ }^{1}$ CNRS, UPR 288 Laboratoire dEnergtique Molculaire et Macroscopique, Combustion (EM2C), \\ Grande Voie des Vignes, 92295 Châtenay-Malabry, France \\ ${ }^{2}$ Ecole Centrale Paris, Grande Voie des Vignes, 92295 Châtenay-Malabry, France
}

\begin{abstract}
In a swirl-stabilised liquid fuel burner, the fuel spray response to the Precessing Vortex Core (PVC) and air flow rate modulations is analysed in non-reacting conditions. A siren-like device is used to modulate the air flow rate at a frequency corresponding to longitudinal combustion instability oscillations observed during reacting tests. Time-resolved Mie scattering images of the fuel spray are recorded and treated with multiple post-processing methods based on Dynamic Mode Decomposition. The spray velocity fluctuations induced by the PVC and the siren-generated modulations are extracted from noisy datasets and studied. The evolution of the PVC impact on the spray for different levels of flow rate fluctuations is followed and a nonlinear interaction mode is highlighted for several intensities of flow rate modulations. It is shown that increasing the flow rate modulations tend to weaken the PVC impact on the spray, progressively disturbing its structure, starting from the downstream part and progressing upstream. These observations on the fuel spray can be used to understand and interpret data obtained in reacting conditions, for example when competition between PVC and longitudinal combustion instabilities occurs.
\end{abstract}

\section{INTRODUCTION}

Legal regulations on the pollutant emissions of gas turbine plants or aircraft engines become increasingly stringent because of growing environmental concerns. Despite its advantages in terms of safety and stability, the use of traditional diffusion flames in gas turbines is hindered by high levels of nitrogen ox- ides (NOx) or carbon monoxide (CO) emissions. Among numerous solutions, Lean Premixed Prevaporized (LPP) regimes can help in reducing flame temperature and thus the emission of thermal NOx by providing a more or less uniform lean mixture of fuel and air [1]. However, when operating in lean conditions, gas turbines often exhibit strong combustion dynamics that may lead to stability issues such as combustion instabilities, flashback or blowout $[2,3]$. In particular, high amplitude self-sustained fluctuations may happen from the coupling between heat release and acoustic oscillations $[4,5]$. These interactions are known to be more easily triggered in premixed or partially premixed conditions [6].

In gas turbines, swirling flows are used to perform an aerodynamical flame stabilisation. However, strongly swirling flows can often exhibit a large scale helical structure, known as a Precessing Vortex Core (PVC) which can disturb the combustion process [7-10]. Studies show that the PVC can be destroyed when the flow is acoustically forced upstream of the swirler [11,12]. A similar scenario is envisaged in Providakis et al. [13] to explain the disappearance of the PVC when the operating point presents a strong longitudinal thermo-acoustic instability.

Johchi et al. [14] recently found a decrease in the PVC signal from the flame and the spray as the global equivalence ratio is increased. This increase of the equivalence ratio is associated with an increase in the intensity of a thermo-acoustic instability. The present study, based on the same burner geometry, is aimed at analysing, in similar but non-reacting conditions, the fuel spray response to the PVC and air flow rate modulations induced by 
a siren-like device. Since the spray of liquid fuel evaporates and then burns, its dynamics strongly impact the flame behaviour and thus must be studied thoroughly.

\section{EXPERIMENTAL SETUP \\ Swirl-stabilised burner}

The BIMER experimental setup $[13,15]$ is equipped with a multi-injection staged injector based on an industrial geometry where fuel and air can mix. The mixture then flows into a rectangular combustion chamber $\left(500 \times 150 \times 150 \mathrm{~mm}^{3}\right)$ at atmospheric pressure with two side silica walls for optical access and three stainless steel water-cooled walls at the top, bottom and entrance of the chamber. Air is controlled by a mass flow controller (Bronkhorst In-Flow) and is preheated (Sylvania SureHeat Max) to enhance fuel vaporization. It then flows through a plenum before being divided between the two stages of the injector. Schematics of the combustion chamber and of the injector are shown on Fig. 1.

The upstream stage, called the pilot stage, contains an 18vane swirler that is designed to allow around $15 \%$ of the global air flow rate to flow through this stage. It is associated with a controlled fuel flow (Bronkhorst Mini Cori-Flow) in a central injection nozzle. This pressurized nozzle (MMP 6390-0-0-03) can inject up to $6.31 . \mathrm{h}^{-1}$ of dodecane (flow number: $1.41 . \mathrm{h}^{-1} \cdot \mathrm{bar}^{-1 / 2}$ ), creating a hollow cone of droplets with a $30^{\circ}$ half-angle.

The downstream stage, called the take-off stage, contains a 20 -vane swirler through which the remaining $85 \%$ of the air flows. The fuel, controlled by a Coriolis flowmeter (Bronkhorst Cori-Flow), is injected through a multipoint device with ten equally spaced holes of $0.3 \mathrm{~mm}$ in diameter placed at the center of the exit of one swirler vane out of two to ensure proper droplet atomization.

The swirlers of each stage are co-rotating and designed to provide a geometrical swirl number close to 1 . The expanding swirling flow in the confined geometry of the chamber creates two recirculating zones which are outlined on Fig. 1: the Inner Recirculation Zone (IRZ) and the Outer Recirculation Zone (ORZ). These outlines come from PIV measurements with solid particle seeding, described with more details in T. Providakis $\mathrm{PhD}$ thesis [16].

In order to ensure a good repeatability between the experiments, the flowmeters are controlled through a in-house LabView software with a National Instruments PCI-6259 card.

In the framework of this non-reacting study, the multipoint fuel injection is not used (pilot-only conditions) because the flow dynamics showed no dependency on the fuel injection mode. Furthermore, to enable proper comparisons with Johchi et al. [14], similar injection conditions are replicated. For this reason, a global equivalence ratio of 0.5 has been chosen with an air flow rate of $32.3 \mathrm{~g} . \mathrm{s}^{-1}$. Air is preheated at $473 \mathrm{~K}$. This operating point was chosen in [14] because it shows a good response to the

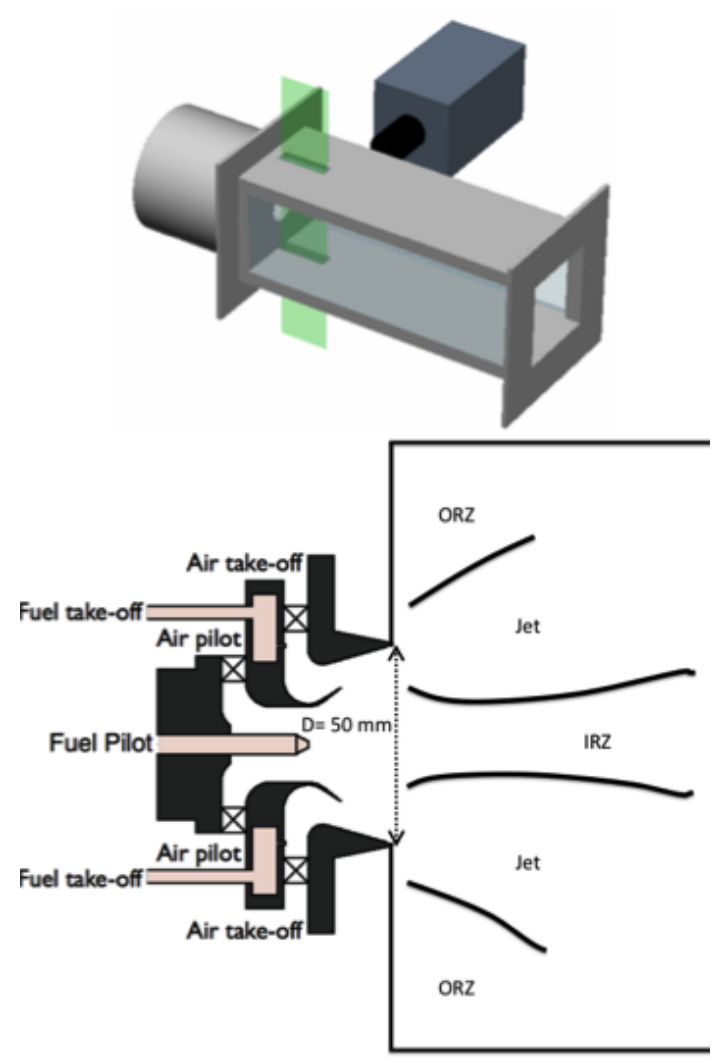

FIGURE 1. SCHEMATICS OF THE EXPERIMENTAL SETUP INCLUDING LASER SHEET (TOP) AND RADIAL CUT OF THE INJECTION DEVICE POSITIONED AT THE ENTRANCE OF THE COMBUSTION CHAMBER SHOWING THE TWO FUEL INJECTION STAGES AND THEIR RESPECTIVE SWIRLERS (BOTTOM). FLOW FROM LEFT TO RIGHT.

PVC. Power and pressure scaling aside, such pilot-only conditions are not unusual in real jet engines and are used when low power is needed (idle, taxi and up to $30 \%$ of the rated thrust).

\section{Siren device}

In order to generate flow rate modulations, an air flow pulsator of type siren with tunable frequency and variable amplitude from the company Combustion Bay One (CBOne, Graz, Austria [17]) is installed in the air feeding line upstream the plenum. The air flows through a $12 \mathrm{~mm}$ diameter nozzle in front of which a wheel with ten 9 mm-high teeth rotates. By alternatively covering and uncovering a precise part of the nozzle, this toothed wheel modulates the flow at a frequency depending on its rotational speed. Since the generated harmonics are quickly damped, the air flow modulation can be considered almost sinusoidal when it reaches the injection device. The center of the toothed wheel can furthermore be vertically moved in order to 


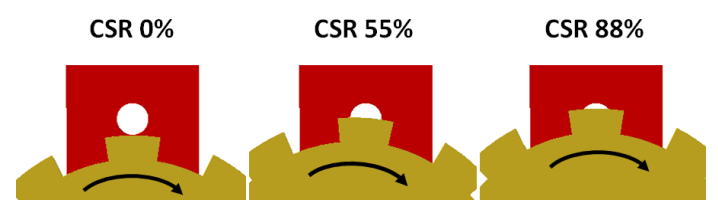

FIGURE 2. RELATIVE POSITIONS OF THE TOOTHED WHEEL AND SIREN NOZZLE FOR DIFFERENT CSR (0\%, 55\% AND 88\%).

control the amount of blocked area of the nozzle. This setup thus gives independent control on the frequency and amplitude of the flow modulations.

The device is controlled by LabView routines and provides analog outputs for the wheel speed and position. Further details on the system can be found in [17].

In the framework of this study, the siren is operated at a fixed frequency of $310 \mathrm{~Hz}$ as it corresponds to the average frequency of the thermo-acoustic instability encountered in reacting conditions for this operating point [14]. The amplitude is however varied to analyse how its interaction with the PVC evolves. To properly define this amplitude, the Covered Surface Ratio (CSR) is introduced as the ratio between the maximum area of the nozzle covered by the tooth and the nozzle area when the tooth is not in front of it. For example, a CSR of $55 \%$ means that $55 \%$ of the nozzle area is alternatively covered and uncovered by the tooth while the remaining $45 \%$ undergoes no modulation. The relative positions of the nozzle and the toothed wheel for different CSR can be seen on Fig. 2.

\section{Diagnostics and post-processing methods}

Since the study is aimed at understanding the effects of the dynamics of the flow field on the fuel spray, time-resolved images of Mie scattering from the fuel droplets are chosen as the main diagnostic. A high-speed camera (Photron Fastcam SA-5) is used and equipped with a $50 \mathrm{~mm}$ lens and a $532( \pm 10) \mathrm{nm}$ filter. It is focused on a laser sheet placed vertically on the axial-radial plane of the chamber (see Fig. 1) and records a $161 \times 119 \mathrm{~mm}^{2}$ region with $704 \times 520$ pixels. However, since the laser sheet is about $90 \mathrm{~mm}$ wide, the useful part of the frame is only $404 \times 520$ pixels with a resolution of $0.23 \mathrm{~mm}$ per pixel. The laser sheet is made by two Quantronix Nd:YAG lasers firing at $10 \mathrm{kHz}$ each with a delay of $25 \mu$ s between two pulses while the camera records at a synchronised frequency of 20000 frames per second for 1.5 seconds. The recorded images can be either directly analysed or treated with a Particle Image Velocimetry (PIV) algorithm.

The PIV procedure is performed by the Dantec Dynamic Studio software using an adaptive correlation algorithm with 2 refinement steps. The final interrogation window size is $16 \times 16$ pixels with $50 \%$ overlap. The PIV resolution is thus $3.6 \mathrm{~mm}$ with an inter-vector spacing of $1.8 \mathrm{~mm}$ and the final velocity map is $49 \times 64$ vectors. Because this procedure is performed on fuel droplets images, it must be kept in mind that the results are not representative of the air flow but highlight the behaviour of the fuel spray.

In order to extract the dynamics from the raw Mie scattering images or from the computed velocity fields, Dynamic Mode Decomposition (DMD) [18] is used in this study. DMD is a mathematical tool which enables to decompose experimental or numerical data into a sum of mono-frequential modes. Although it seems quite close to the Proper Orthogonal Decomposition (POD) used in some PVC studies [12, 19, 20], DMD provides interesting properties for the present case. First, since both the siren-induced modulations and the PVC are spectrally narrow phenomena without strong harmonics, they appear in two separate modes. They can thus be independently analysed whereas POD might have given modes that include both phenomena (as in [19], for instance). Another advantage of the DMD is the fact that it provides information on the temporal evolution of the data which can help in assessing the stationarity of the process. However, this technique remains quite new and strong links between DMD modes and physical structures remain to be fully established. For a further understanding, comparisons between POD and DMD results can be found in [21].

To perform a DMD analysis, time-resolved data are seen as sequences of $N s$ snapshots which are placed into two matrices, $V_{1}^{N s-1}$ and $V_{2}^{N s}$, respectively containing snapshots from the first one to the penultimate and from the second one to the last. Each column of the matrices regroups the data contained in one snapshot (which can be a mix of any diagnostic: velocity, pressure, Mie intensity... [22]) and the lines correspond to the time evolution. If a linear mapping $A$ is assumed between the snapshots, the following link is obtained:

$$
V_{2}^{N s}=A V_{1}^{N s-1}
$$

Obtaining the dominant dynamical features of the analyzed phenomenon consists in computing the eigenvalues and eigenvectors of $A$. However, since $A$ cannot be directly computed from the datasets, the DMD aims at obtaining a companion matrix $S$ which can approximate the eigenvectors and eigenvalues of $A$. This matrix is defined as the best one to approximate the following equation:

$$
V_{2}^{N s}=V_{1}^{N s-1} S
$$

The matrix $S$ is obtained through a QR-decomposition of $V_{1}^{N s-1}$ and the diagonal matrix of its eigenvalues $D$ associated with the eigenvectors matrix $X$ can be computed. The matrix of dynamic modes $M_{d m}$ can then be extracted by the operation $M_{d m}=V_{1}^{N s-1} X$. The imaginary part of an eigenvalue can be 
linked to the frequency of the associated mode and the real part represents its growing or decaying behaviour. The DMD spectrum is obtained at this step by plotting the norm of the dynamic modes versus their respective frequencies but DMD can also be used to perform a filtering operation and keep only one or a few modes to reconstruct the dataset. Indeed, equation (2) can be modified to introduce the matrices $X$ and $D$ :

$$
V_{2}^{N s}=V_{1}^{N s-1} S=V_{1}^{N s-1} X D X^{-1}
$$

In the diagonal matrix of the eigenvalues, replacing an eigenvalue by a zero results in suppressing the associated mode in the reconstruction. One can then keep only the interesting eigenvalues in a diagonal matrix $D_{f}$ and perform a filtered reconstruction:

$$
V_{f 2}^{N s}=V_{1}^{N s-1} X D_{f} X^{-1}
$$

The reconstructed dataset then represents the effects of the modes associated to the kept eigenvalues in $D_{f}$, enabling to perform a quantitative filtered reconstruction of the data.

\section{NON-MODULATED SPRAY CHARACTERIZATION}

In this part, PIV post-processing is performed on the Mie scattering images to obtain spray velocity fields. They can be used to gather information on the encountered dynamics. These velocity fields are processed with DMD to obtain mean values or highlight particular modes.

\section{Spatial distribution of the spray}

As stated before, the objective of the present paper is to study the spray response to PVC as well as flow oscillations induced by the siren. The fuel droplets are used for the PIV postprocessing, meaning that only spray velocity maps are obtained. They do not necessarily represent the underlying air flow because the spray is polydisperse and the droplets are on average larger than classical oil seeding ones which leads to large Stokes number variations. However, spray velocities remain interesting to analyse because, in reacting conditions, fuel droplets eventually vaporize and burn and thus their dynamics strongly impact the flame. Nevertheless, fuel injection is highly non uniform and it is needed to assess its spatial extent to avoid inaccurate interpretations of the PIV results.

To perform this analysis, Mie scattering images with the background substracted are binarised using a threshold corresponding to 1.1 times the highest pixel value in known black areas outside of the laser sheet. Pixels with higher values can be considered as "lit" and are likely to stem from the presence of a fuel droplet. The PIV windows position and size are then used to count the number of lit pixels in each of them. A window

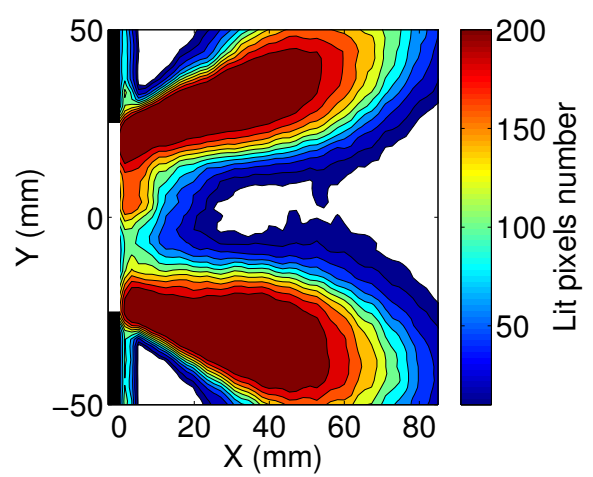

FIGURE 3. CONTOUR PLOT (STARTING AT 10) OF THE AVERAGE NUMBER OF LIT PIXELS PER PIV WINDOW (CSR 0\%). FLOW FROM LEFT TO RIGHT.

with a high number of lit pixels is likely to give a proper PIV result thanks to a good local droplet density. A map of the average number of lit pixels per PIV window for a run where the siren was turned off can be found on Fig. 3 .

The map shows two symmetrical arms with a high number of lit pixels. They correspond to the main spray area with a high droplet density. The PIV results in this region are thus likely to be reliable. However, the average number of lit pixels in the PIV windows drops drastically in the center part of the image and on the top and bottom regions (corresponding to the recirculation zones). The PIV data obtained in these areas are likely to present gaps and the results have to be treated very carefully to avoid misinterpretations. The low number of lit pixels in these regions can be explained by the fact that the fuel droplets must spend a relatively long time in the combustion chamber before appearing in the recirculation zones: because the air is preheated at $200^{\circ} \mathrm{C}$, the smaller droplets following the air flow are thus likely to be already vaporised while most of the bigger ones are not able to enter these zones due to ballistic effects [23]. When the siren is turned on and operated at its maximum amplitude (CSR 88\%), the map of the average number of lit pixels per PIV window is extremely similar to the one shown on Fig. 3. The only difference between the two lies in the center part of the image which presents a bit more lit pixels in the modulated case.

\section{Mean flow characterization}

To obtain a mean velocity field the classical method would be to perform a time-averaging operation on the velocity snapshots. However, to be as close as possible to the post-processing performed to extract the PVC and siren fluctuations, the method used here is different. First, the velocity field snapshot sequence is decomposed with a DMD algorithm. It is then reconstructed by keeping only the zero frequency mode of the spectrum (that is the mode associated to an eigenvalue with no imaginary part). 

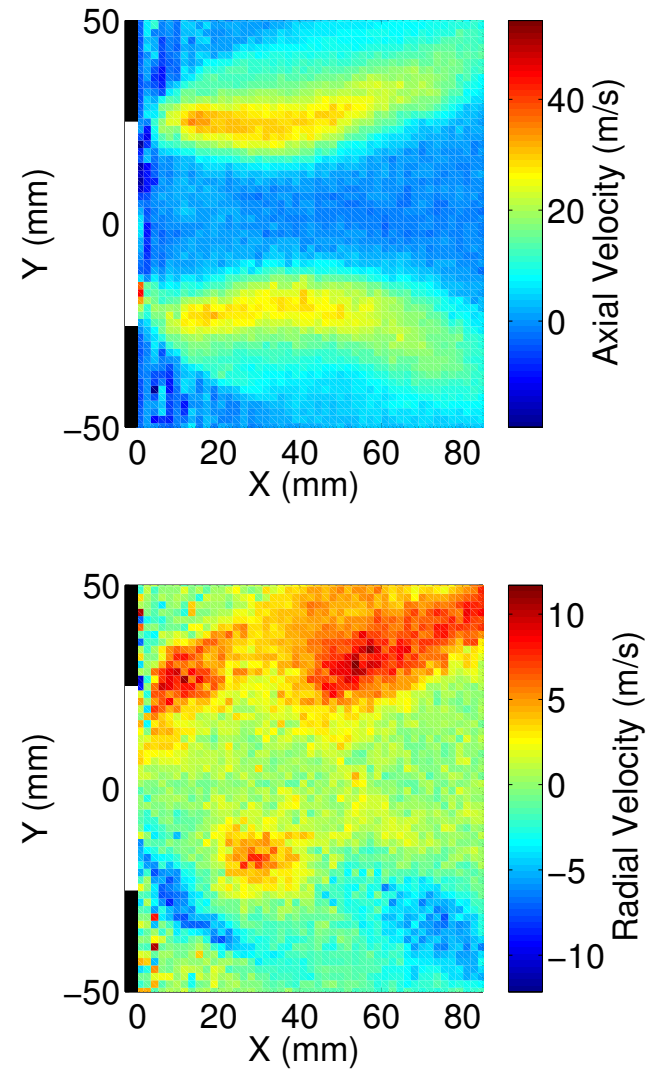

FIGURE 4. MEAN AXIAL (TOP) AND RADIAL (BOTTOM) SPRAY VELOCITY FIELDS FROM A RECONSTRUCTION OF THE DATA BASED ON THE ZERO FREQUENCY MODE OF THE DMD (CSR 0\%). FLOW FROM LEFT TO RIGHT.

The obtained sequence is finally time-averaged to get rid of its growing or decaying behaviour (real part of the eigenvalue). The DMD operation acts as a filtering tool to remove noise and the resulting "DMD-average" velocity field differs from the classical average velocity field by less than $10 \%$ in the regions of interest (in comparison, the RMS value in the same regions is around $30 \%$ of the mean one). Although unusual, this method is chosen for reason of consistency with the following fluctuation studies where the denoising provided by the DMD proves to be very useful.

The average axial and radial velocities can be seen on Fig. 4. They result from the treatment of 501 PIV snapshots from a run where the siren was turned off. It is relevant to indicate that the same analysis on a run with the siren working at its maximum amplitude gives similar fields, meaning that amplitude fluctuations do not modify the mean air flow rate and spray mean behaviour.
The mean axial velocity field is typical of a swirling injector with a conical jet region diverging from the axis of the chamber. The velocities in this region are around $30 \mathrm{~m} \cdot \mathrm{s}^{-1}$ which is consistent with results obtained for higher flow rates [13]. Even though the seeding is not optimal there, a central zone with negative velocities due to vortex breakdown can be found, surrounded by the jet region: the Inner Recirculation Zone (IRZ). On the top and bottom parts of the chamber, the Outer Recirculation Zone (ORZ) also shows negative velocity values. These zones are of paramount importance for aerodynamical flame stabilisation by recirculating part of the hot burnt gases upstream.

A look at the mean radial velocity field shows that the spray is mainly diverging with velocities lower than $10 \mathrm{~m} . \mathrm{s}^{-1}$ but, and it is especially visible on the bottom half here, a region exists where the spray contracts itself $(X \approx 30 \mathrm{~mm})$ which can also be highlighted by the shape of the jet region on the axial velocity field. Overall, the recirculation zones show weak radial velocities.

\section{Precessing Vortex Core}

Before studying the influence of flow rate modulations on the impact of the Precessing Vortex Core on the spray, it is first necessary to analyse the effect of the PVC alone on the fuel droplets velocity. The PVC is often studied by performing a phase-averaging operation on PIV fields. This however requires either a really steady phenomenon with a known constant frequency or a clean synchronising signal responding to the studied phenomenon both of which being not available in this study. Furthermore, and probably because the seeding based on the fuel droplets is not perfect, the PVC signature in the PIV fields is hindered by the noise and direct phase-averaging attempts ended up unsuccessful.

To overcome these issues, Dynamic Mode Decomposition is used as a denoising tool before carrying out the phase-averaging operation. The snapshot sequence is decomposed and the mode associated with the PVC is identified as the highest peak in the spectrum between 1550 and $1750 \mathrm{~Hz}$ (the PVC frequency for this operating point being around $1650 \mathrm{~Hz}$ ). The snapshot sequence is then reconstructed with only this mode and a phase-averaging operation is performed. Since the frequency is now fixed and known, the phase-averaging is easy to implement and enables to get rid of the growing or decaying behaviour of the mode. A test of this method has been performed on synthetic signals prior to its application here and it gave accurate quantitative results.

The axial and radial droplet velocity fields close to the injector can be seen on Fig. 5 for an arbitrary phase angle (chosen so that the fluctuations are the highest) of the reconstructed data with the PVC mode. They stem from the analysis of 501 instantaneous velocity fields from a run where the siren was turned off to avoid any disturbing phenomenon. This number of snapshots may seem quite low but it comes from a balance between two 

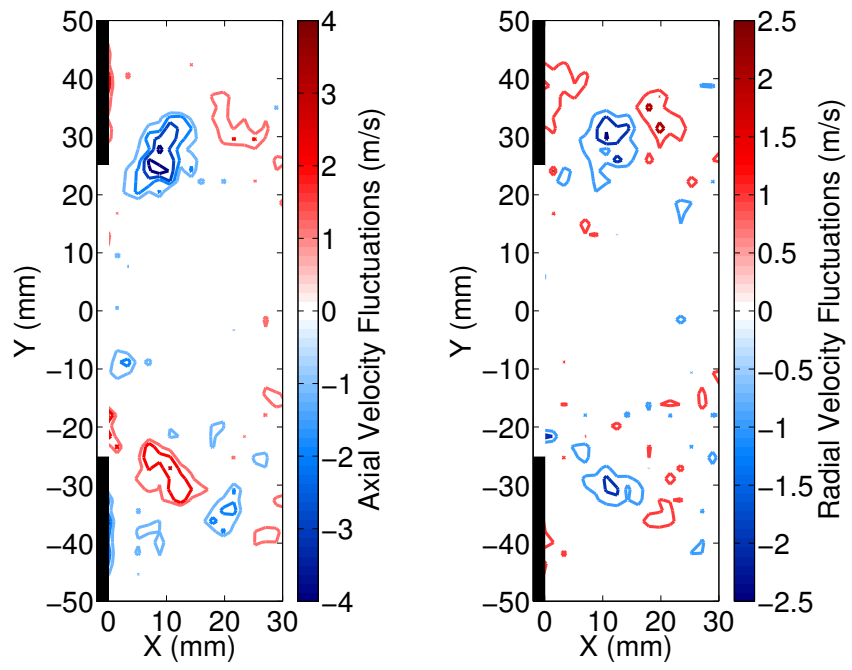

FIGURE 5. SPRAY VELOCITY FLUCTUATIONS FROM A RECONSTRUCTION OF THE DATA BASED ON THE PVC MODE OF THE DMD (CSR 0\%). LEFT: AXIAL COMPONENT. RIGHT: RADIAL COMPONENT. FLOW FROM LEFT TO RIGHT.

noise sources. When long sequences are used, noise is usually statistically reduced. However, since the PVC frequency fluctuates a bit with time and the number of DMD modes obtained from the decomposition depends on the number of used snapshots, performing a DMD on long sequences leads to an artificial spread of the PVC fluctuations on several modes. The value of 501 snapshots is thus chosen as it enables to keep the PVC on only one mode while also keeping a sufficient duration to reduce noise. The detected frequency for this mode is $1651 \mathrm{~Hz}$ and it is to be noted that only the first half of the field on the x-axis shows coherent structures, the second half (not shown here) being mainly noise.

On the droplet axial velocity field, antisymmetrical structures of perturbations up to $4 \mathrm{~m} . \mathrm{s}^{-1}$ can be observed. For higher phase angles, these structures are convected downstream until the end of the cycle. On the radial velocity field, it can be seen that both arms of the spray move in the same direction for the same axial position with velocities around $2 \mathrm{~m} . \mathrm{s}^{-1}$ (particularly visible for $\mathrm{X} \approx 10 \mathrm{~mm}$ ). As these structures are also convected downstream with the progress in the cycle, they tend to give a snake-like motion to the image of the spray in this plane which can be seen as the trace of a rotating helical structure in a radial cut.

\section{SPRAY EVOLUTION WITH THE MODULATION Characterization of the siren-generated oscillations}

To perturb the Precessing Vortex Core, the flow rate modulations created by the siren are used and their impact on the spray
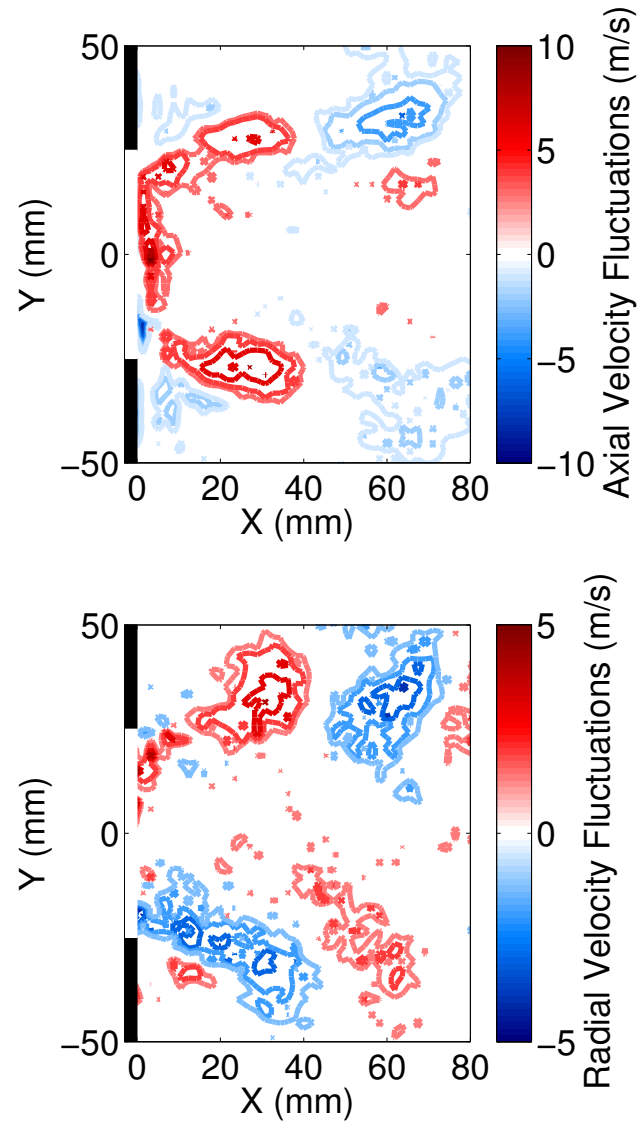

FIGURE 6. SPRAY VELOCITY FLUCTUATIONS FROM A RECONSTRUCTION OF THE DATA BASED ON THE SIREN MODE OF THE DMD (CSR 88\%). TOP: AXIAL COMPONENT. BOTTOM: RADIAL COMPONENT. FLOW FROM LEFT TO RIGHT.

must thus be characterized. To characterize the modulation, the siren is operated at $310 \mathrm{~Hz}$ and at its maximum amplitude which leads to the toothed wheel covering $88 \%$ of the nozzle surface (i.e. a CSR of $88 \%$ ).

The treatment performed on the velocity fields is the same as for the study of the PVC effect on the spray. The mode that is kept to reconstruct the snapshot sequence corresponds to the peak of the spectrum between 260 and $360 \mathrm{~Hz}$. It is to be noted that there are less issues and noise than for the PVC study since here the amplitude of the fluctuations is higher. The resulting axial and radial velocity fields for an arbitrary phase angle can be seen on Fig. 6.

The droplet axial velocity field is characterized by its symmetry and the presence of structures along the axial direction. These structures represent the droplet axial velocity variations (up to $10 \mathrm{~m} . \mathrm{s}^{-1}$ ) induced by the successive presence of the teeth of the toothed wheel in front of the siren nozzle. As the cycle progresses, these structures are convected downstream. The 
fluctuations near the axis of the upstream part of the chamber ( $\mathrm{X}<10 \mathrm{~mm}, \mathrm{Y} \approx 0 \mathrm{~mm}$ ) are quite high. This leads to think that the upstream part of the IRZ is strongly affected by the sireninduced modulations, maybe up to the cancellation of the reversed flow. The radial velocity field shows moving structures which highlights the fact that the spray is alternatively expanding and contracting (with velocities up to $5 \mathrm{~m} . \mathrm{s}^{-1}$ ). The expansions are correlated with positive axial velocity fluctuations whereas the contractions correspond to negative fluctuations (see $\mathrm{X} \approx 30$ and $60 \mathrm{~mm}$ ). This stems from the fact that the flow rate modulations are generated by the siren before the swirlers. An increase in mass flow rate in the swirlers leads to an increased azimuthal air velocity which in turn leads to a stronger centrifugal effect on the fuel droplets and then to a radial expansion of the spray. The siren-generated waves are thus not entirely axial but also present a radial component.

As stated previously, the PVC impact on the data after the PIV operation is quite low and performing a similar study of the PVC from the spray velocity fields with the siren turned on would produce mainly noisy results. To analyse the impact of the PVC on the spray and its evolution with the siren-generated fluctuations, it is decided to use the raw data and perform the analyses directly on the Mie scattering images. The Mie scattering intensity depending on both the droplets size and density, the obtained results are not quantitative but provide useful qualitative insight on the spray behaviour and on the liquid fuel repartition.

\section{Evolution of the spray dynamics with the modulation amplitude}

When the siren is turned on, the spray shows varying responses to the PVC depending on the amplitude of the siren modulation. For seven different amplitudes (CSR 0, 20, 37, 55, 67, 77 and $88 \%$ ), DMDs are performed on the Mie scattering images to determine the dynamical features of the spray. To avoid high computational costs since the whole Mie scattering image is used, a binning of $4 \times 4$ pixels is performed before the DMD computations.

For a CSR of 55\%, the DMD spectrum (based on 501 samples) shown on Fig. 7 enables to highlight the three main phenomena that are followed in this section. The first peak around $310 \mathrm{~Hz}$ represents the effect of the siren-generated modulations. The second peak around $1650 \mathrm{~Hz}$ is the result of the PVC acting on the droplets. The third peak around $1960 \mathrm{~Hz}$ is also very interesting as it corresponds exactly to the sum of the frequencies of the two previous ones. This means that the PVC and the siren-induced fluctuations do not only coexist but also interact with each other. A similar non-linear interaction between a longitudinal thermo-acoustic oscillation and a PVC has already been highlighted in the flame response signals in previous studies $[11,19,20]$.

To follow the evolution of these three features, it is necessary

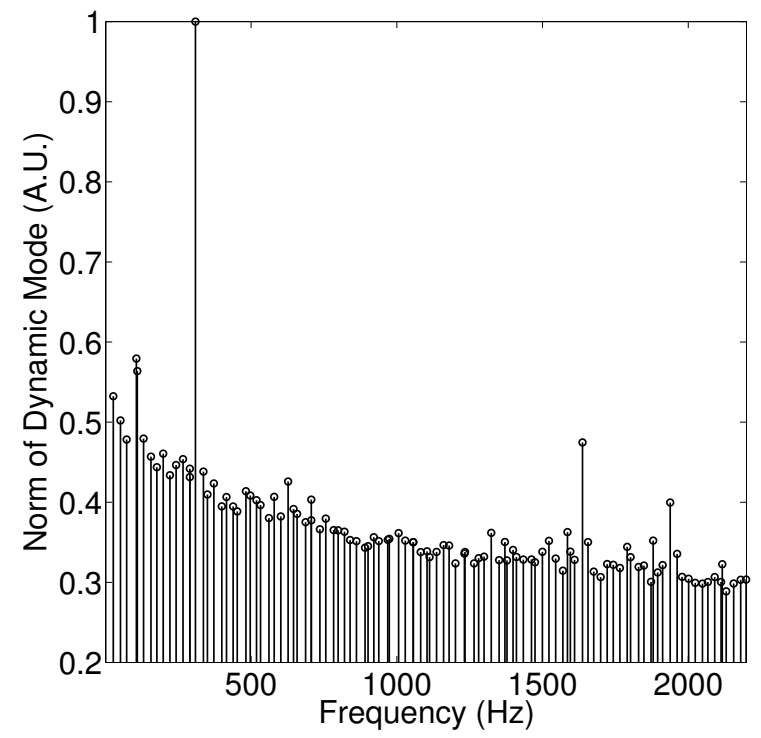

FIGURE 7. DMD SPECTRUM FOR A $55 \%$ CSR SHOWING THREE MAJOR FEATURES.

to measure their respective intensities. A novel approach, previously proposed in [24] with encouraging results, is to compute a Signal-to-Noise Ratio (SNR) coefficient associated with the chosen peak on the DMD spectrum. This is performed by dividing the peak value by the median of the values within an interval containing the peak. The chosen intervals are $[260 ; 360] \mathrm{Hz}$ for the siren peak, $[1550 ; 1750] \mathrm{Hz}$ for the PVC peak and $[1860$; 2060] $\mathrm{Hz}$ for the interaction peak. The first interval is narrower than the two other ones to avoid the deterrent influence of the high values in the low-frequency region of the spectrum.

Since each laser fires at $10 \mathrm{kHz}$ and the camera records at $20000 \mathrm{fps}$, each recording can be seen as two intertwined $10 \mathrm{kHz}$ sets of data. Each of these sets then undergoes 30 DMDs with 501 samples each to cover the full extent of the recording time and, for each DMD spectrum, the three SNR corresponding respectively to PVC, siren and interaction modes are computed. A statistical analysis can then be performed on these SNR to obtain their mean value with the related $95 \%$ confidence interval for each of the 7 successive CSR values. The evolution of these SNR with the CSR (ie the amplitude of the siren-induced fluctuations) is plotted on Fig. 8. Since SNR computations on noisy parts of the spectrum give results around 1.1, this value has been chosen as a threshold to assess the viability of the plotted points.

When the CSR is 0\%, the siren generates no modulation of the air flow. This explains why only the PVC SNR is above the threshold. As the CSR increases, the siren SNR also increases as expected and the PVC SNR decreases. This is consistent with the results of Moeck et al. [11] and with what was observed in react- 


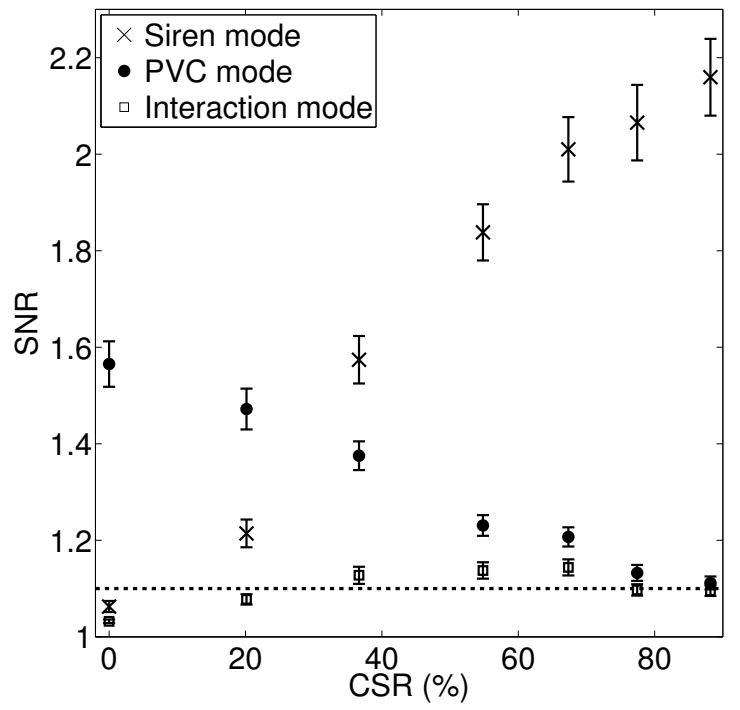

FIGURE 8. SNR MEAN VALUE AND 95\% CONFIDENCE INTERVAL FOR THE THREE MAIN FEATURES VERSUS COVERED SURFACE RATIO. DOTTED LINE: 1.1 VIABILITY THRESHOLD

ing conditions where increasing the equivalence ratio increased the thermo-acoustic oscillations and decreased the PVC impact on the spray [14]. Here, for a CSR of $88 \%$, the PVC cannot be detected anymore. The SNR associated with the interaction shows an interesting trajectory since it is above the threshold only when the PVC and the siren are both high enough, which means that the interaction exists only for a few specific conditions, namely when the flow rate modulations are high enough to impact the PVC but still too low to destroy it.

\section{Spatial evolution of the PVC impact on the spray}

Following the evolution of the SNR gives data on the relative siren and PVC impact strength on the spray but does not provide any information on the spatial evolution of the structure of the PVC disturbance of the spray. To obtain this information, it is first needed to observe the spatial effect of the PVC on the spray without any siren-induced modulations as a reference point. The PVC impact being antisymmetric and limited to the first few centimetres of the spray, DMD has been performed on a small region of interest of the Mie scattering images only $(120 \times 120$ pixels, see the smaller image on Fig. 9). Because of this reduced region of interest, no pixel binning is needed to reduce computational costs and the DMDs are now performed on data with native resolution. A resulting mode (from 501 snapshots) at the PVC frequency for a CSR of $0 \%$ can be seen on Fig. 9.

This mode shows structures that are convected downstream as the PVC cycle progresses, meaning that the Mie scattering

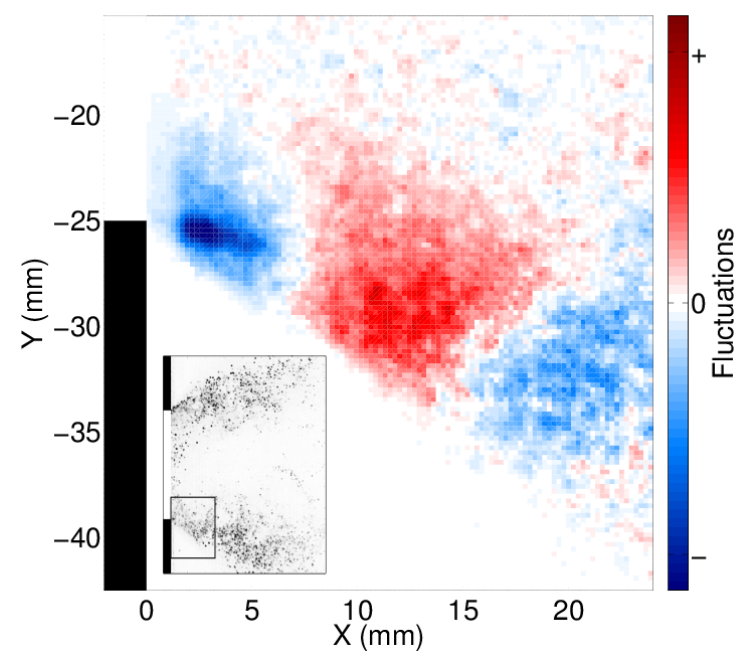

FIGURE 9. REFERENCE MIE SCATTERING DMD MODE (CSR 0\%) USED FOR THE COMPUTATION OF THE CORRELATION COEFFICIENTS. THE SMALLER IMAGE SHOWS THE POSITION OF THE REGION OF INTEREST IN THE FULL SIZE MIE SCATTERING IMAGE. FLOW FROM LEFT TO RIGHT.

signal, that is the droplet sizes and/or density, is perturbed by the PVC. This leads to an uneven liquid fuel distribution which is likely to modulate the flame in reacting conditions. This mode is arbitrarily chosen as a reference of the undisturbed PVC impact on the spray and all the following results are obtained with comparisons to this mode.

To analyse if these PVC-induced spray structures are disturbed by the flow rate modulations, $2 \mathrm{D}$ cross-correlation coefficients are computed. Similarly to what was done for the SNR computations, 60 decompositions are performed for each CSR value. The obtained PVC modes for each DMD are then compared to the reference mode by calculating a cross-correlation coefficient. However, the analysed DMD mode and the reference mode can differ by their phase which has to be accounted for. This phase difference is corrected by phase-shifting the analysed mode until the cross-correlation coefficient between the two images is the highest. The value is then used to compute an average correlation coefficient and a $95 \%$ confidence interval for each CSR. The resulting plot can be seen on Fig. 10.

First, for a CSR of $0 \%$, the mean value of the normalized correlation coefficient is around 0.85 . Although this value is not as high as one would expect, it must be kept in mind that the reference mode shows noisy parts that have a detrimental effect on the global correlation coefficient. It can thus be considered that the arbitrarily chosen reference mode is quite representative of the average undisturbed PVC impact on the spray (which will be confirmed by the local correlation values found on Fig. 11). For 


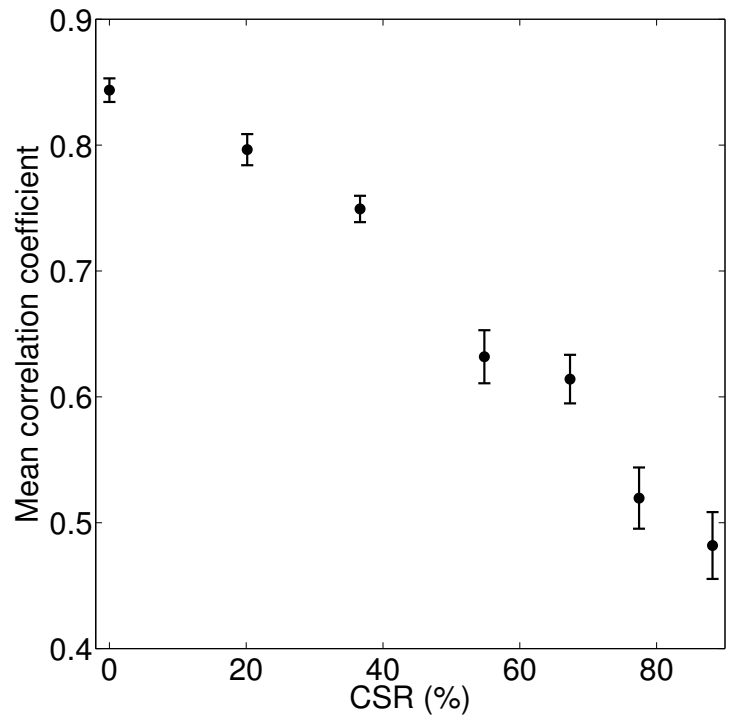

FIGURE 10. MEAN VALUE AND 95\% CONFIDENCE INTERVAL OF THE CORRELATION COEFFICIENT WITH THE PVC REFERENCE MODE VERSUS COVERED SURFACE RATIO.

the higher CSR values, it can be seen that the mean correlation coefficient decreases as the CSR increases, showing that the PVC impact on the spray in the modulated cases increasingly differs from the undisturbed case as the modulations get stronger. This tendency is the same as for the SNR plotted on Fig. 8 but the information provided is different. The SNR plot shows that the impact of the PVC on the spray is getting weaker with increasing flow rate modulations whereas the correlation coefficient plot does not rely on the strength of the mode but shows that its spatial structure is disturbed by the flow rate modulations.

For a further understanding, it is necessary to determine where the spatial structure of the PVC impact on the spray is the most perturbed by the effect of the siren-induced modulations. For this purpose, maps of local cross-correlation coefficients are computed. The image for each mode is divided into $32 \times 32$ pixels windows with $75 \%$ overlap and the $2 \mathrm{D}$ cross-correlation coefficients between the windows of the analysed mode and the windows of the undisturbed reference mode (Fig. 9) are computed. As before, these values are averaged for each CSR and the resulting maps can be seen on Fig. 11. Since the PVC is really weak for CSR $88 \%$, the associated map is not shown because it does not provide any relevant information.

When no flow rate modulation is generated by the siren (CSR 0\%), the local correlation coefficient is high in the region where the spatial structures are located and lower outside (see Fig. 9). However, in the downstream part of the spray, the correlation coefficients are lower than in the upstream part. It means that, even without air flow rate modulations, the PVC induces a more defined and stable effect on the spray closer to the injector and gets less defined downstream. For higher CSR values, when the CSR increases, the local correlation coefficients relatively to the undisturbed reference mode decrease and start reaching low values in the downstream part of the spray, showing that this region gets even more perturbed to the point where no PVC impact can be identified. This loss of structuration then progresses upstream until it reaches the beginning of the spray. The PVC impact on the spray is thus observed on an increasingly shorter distance as the mass flow rate modulations increase.

\section{CONCLUSION AND PERSPECTIVES}

The concurrent impacts of the PVC and air flow rate modulation on the fuel spray in a swirl stabilised burner have been studied in non reacting conditions. Air flow rate modulations of different amplitudes are carried out with a siren, at a frequency of $310 \mathrm{~Hz}$. This particular frequency was chosen because it is associated with self-sustained longitudinal oscillations during reacting test combustion instabilities. The siren modulation is assumed to mimic the effect of the instability on the air flow. Timeresolved Mie scattering images of the fuel droplets in a vertical axial-radial plane have been recorded and processed with different methods depending on which phenomena were to be highlighted.

First, with the help of a PIV post-treatment, velocity maps for the spray have been extracted. With the help of a denoising technique based on a DMD pre-processing, mean droplet velocity fields were obtained as well as the droplet velocity fluctuations generated by the impact of the PVC or by the siren modulation when it is turned on. The mean fields show a typical behaviour for a confined swirling flow with a conical jet region coupled with inner and outer recirculation zones. The fluctuations generated by the PVC give a weak but visible helical motion to the spray. By generating fluctuations of mass flow rate upstream of the swirler, the siren changes both the axial and azimuthal air velocity components in the chamber which induces a strong effect on both the radial and axial velocity fluctuations of the spray. This leads to a spray expanding with positive axial velocity fluctuations and contracting with negative ones.

To monitor the evolution of the strength of the PVC on the spray with increasing amplitude of the siren modulation, the raw Mie scattering images have been processed with DMD. As expected, the intensity of the PVC mode (measured by the SNR of the DMD spectrum peak) decreases with increasing amplitude of the modulation. However, for some conditions, a nonlinear interaction mode can appear. To understand how the spatial structure of the PVC evolves with the amplitude of the flow rate modulations, the PVC modes for each case are compared with a reference case when the siren is turned off. Mean 2D cross-correlation coefficients between the undisturbed reference mode and modes computed for different CSR decrease as the 

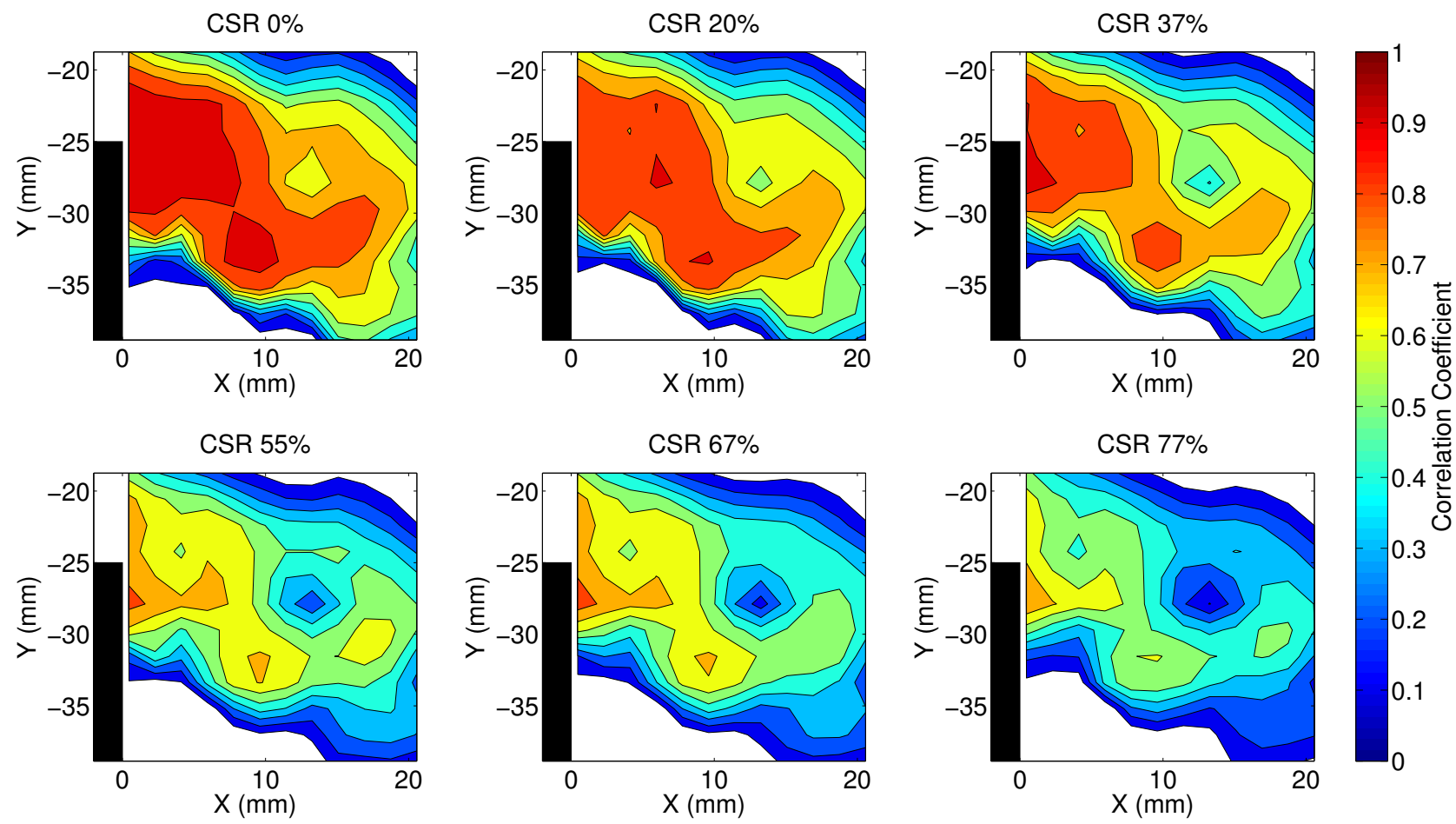

FIGURE 11. MAPS OF AVERAGE LOCAL CORRELATION COEFFICIENTS FOR DIFFERENT CSR. CONTOUR THRESHOLDS GO FROM 0.1 TO 0.9 WITH 0.1 STEPS. FLOW FROM LEFT TO RIGHT.

amplitude of the siren-generated modulations increase. It shows that the spatial structure of the PVC impact on the spray is progressively destroyed by the flow rate modulations. Maps of local cross-correlation coefficients highlight the fact that this distortion starts by the downstream part of the spray and moves upstream as the modulation amplitude is increased leading to a shorter impact of the PVC on the spray.

This study having been conducted on the fuel spray in a non-reacting case, two additional series of experiments are to be performed. First, a cold flow analysis with proper oil seeding will be performed in a near future to study the air flow itself and extract the differences with the spray response. The second step is to conduct similar experiments in reacting cases to fulfill the link with the Johchi et al. dataset [14], especially by highlighting the differences between siren-induced modulations and naturally-occuring thermo-acoustic oscillations. The insight on the acoustic/PVC competition given by such studies would help understanding the stabilisation processes leading to bistable flames in this swirl-stabilised staged burner as observed in [24].

\section{Acknowledgment}

The authors would like to gratefully acknowledge Safran/Snecma for supporting this study. We also thank
Fabrice Giuliani of CBOne for designing, making and helping set up the siren device as well as the technical staff at EM2C laboratory for their help with the enhancement of the experimental setup.

\section{REFERENCES}

[1] Lefebvre, A. H., 1995. "The role of fuel preparation in low-emission combustion”. Journal of Engineering for Gas Turbines and Power, 117(4), Oct., pp. 617-654.

[2] Candel, S., 2002. "Combustion dynamics and control: progress and challenges". Proceedings of the Combustion Institute, 29(1), pp. 1-28.

[3] Huang, Y., and Yang, V., 2009. "Dynamics and stability of lean-premixed swirl-stabilized combustion". Progress in Energy and Combustion Science, 35(4), Aug., pp. 293364.

[4] Krebs, W., Flohr, P., Prade, B., and Hoffmann, S., 2002. "Thermoacoustic stability chart for high-intensity gas turbine combustion systems". Combustion Science and Technology, 174(7), July, pp. 99-128.

[5] Barbosa, S., Scouflaire, P., and Ducruix, S., 2009. "Time resolved flowfield, flame structure and acoustic characterization of a staged multi-injection burner". Proceedings of 
the Combustion Institute, 32(2), pp. 2965-2972.

[6] Ducruix, S., Schuller, T., Durox, D., and Candel, S., 2003. "Combustion dynamics and instabilities: Elementary coupling and driving mechanisms". Journal of Propulsion and Power, 19(5), Sept., pp. 722-734.

[7] Syred, N., and Beer, J. M., 1974. "Combustion in swirling flows: a review". Combustion and Flame, 23(2), pp. 143201.

[8] Froud, D., O'doherty, T., and Syred, N., 1995. "Phase averaging of the precessing vortex core in a swirl burner under piloted and premixed combustion conditions". Combustion and Flame, 100(3), pp. 407-412.

[9] Syred, N., 2006. "A review of oscillation mechanisms and the role of the precessing vortex core (PVC) in swirl combustion systems". Progress in Energy and Combustion Science, 32(2), Jan., pp. 93-161.

[10] Oberleithner, K., Sieber, M., Nayeri, C. N., Paschereit, C. O., Petz, C., Hege, H.-C., Noack, B. R., and Wygnanski, I., 2011. "Three-dimensional coherent structures in a swirling jet undergoing vortex breakdown: stability analysis and empirical mode construction". Journal of Fluid Mechanics, 679, July, pp. 383-414.

[11] Moeck, J. P., Bourgouin, J.-F., Durox, D., Schuller, T., and Candel, S., 2012. "Nonlinear interaction between a precessing vortex core and acoustic oscillations in a turbulent swirling flame". Combustion and Flame, 159(8), Aug., pp. 2650-2668.

[12] Alekseenko, S. V., Dulin, V. M., Kozorezov, Y. S., and Markovich, D. M., 2012. "Effect of high-amplitude forcing on turbulent combustion intensity and vortex core precession in a strongly swirling lifted propane/air flame". Combustion Science and Technology, 184(10-11), Oct., pp. 1862-1890.

[13] Providakis, T., Zimmer, L., Scouflaire, P., and Ducruix, S., 2013. "Characterization of the coherent structures in swirling flames stabilized in a two-staged multi-injection burner: Influence of the staging factor". Comptes Rendus Mécanique, 341(1-2), Jan., pp. 4-14.

[14] Johchi, A., Zimmer, L., and Tanahashi, M., 2014. "Investigation on the acoustic behavior of a turbulent swirlstabilized combustor fed with liquid fuel". In 17th International Symposium on Applications of Laser Techniques to Fluid Mechanics, Lisbon, Portugal.

[15] Providakis, T., Zimmer, L., Scouflaire, P., and Ducruix, S., 2012. "Characterization of the acoustic interactions in a two-staged multi-injection combustor fed with liquid fuel". Journal of Engineering for Gas Turbines and Power, 134(11).

[16] Providakis, T., 2013. "Compétition entre structures aérodynamiques et modes acoustiques dans une flamme swirlée: Influence de la répartition de carburant”. PhD thesis, Ecole Centrale Paris, Apr.
[17] Giuliani, F., Lang, A., Gradl, K. J., Siebenhofer, P., and Fritzer, J., 2012. "Air flow modulation for refined control of the combustion dynamics using a novel actuator". Journal of Engineering for Gas Turbines and Power, 134.

[18] Schmid, P. J., 2010. "Dynamic mode decomposition of numerical and experimental data". Journal of Fluid Mechanics, 656, July, pp. 5-28.

[19] Boxx, I., Stöhr, M., Carter, C., and Meier, W., 2010. “Temporally resolved planar measurements of transient phenomena in a partially pre-mixed swirl flame in a gas turbine model combustor". Combustion and Flame, 157(8), Aug., pp. 1510-1525.

[20] Steinberg, A., Boxx, I., Stöhr, M., Carter, C., and Meier, W., 2010. "Flow-flame interactions causing acoustically coupled heat release fluctuations in a thermo-acoustically unstable gas turbine model combustor". Combustion and Flame, 157(12), Dec., pp. 2250-2266.

[21] Schmid, P. J., Violato, D., and Scarano, F., 2012. "Decomposition of time-resolved tomographic PIV". Experiments in Fluids, 52(6), Feb., pp. 1567-1579.

[22] Richecoeur, F., Hakim, L., Renaud, A., and Zimmer, L., 2012. "DMD algorithms for experimental data processing in combustion". In Proceedings of the Summer Program, p. 459.

[23] Lacour, C., Durox, D., Ducruix, S., and Massot, M., 2011. "Interaction of a polydisperse spray with vortices". Experiments in Fluids, 51(2), Feb., pp. 295-311.

[24] Renaud, A., Ducruix, S., Scouflaire, P., and Zimmer, L., 2014. "Flame shape transition in a swirl stabilised liquid fuel burner". Proceedings of the Combustion Institute, In Press. 